\title{
How differential privacy will affect our understanding of population growth in the United States
}

Alexis R. Santos-Lozada ${ }^{1,2}$

Danilo T. Pérez-Rivera ${ }^{3}$

Aarti C. Bhat ${ }^{1,2}$

1. Department of Human Development and Family Studies, Pennsylvania State University

2. Population Research Institute, Pennsylvania State University

3. Center for Neural Science at New York University

Corresponding Author

* Alexis R. Santos-Lozada

Address: $\quad 102$ Health and Human Development

University Park, PA 16802

Tel. (814) 863-0117

Email: $\quad$ ars39@psu.edu

https://orcid.org/0000-0002-8437-4493

https://orcid.org/0000-0002-0066-6799

https://orcid.org/0000-0002-4385-8969

Classification

Social Sciences

Keywords

Census, Differential Privacy, Disclosure Avoidance, Population Change, Demography

\section{Author Contributions}

A.R.S.L. designed research; A.R.S.L. and D.T.P.R. performed research; A.R.S.L. and D.T.P.R. analyzed data; A.R.S.L. D.T.P.R., and A.C.B. wrote the paper

This PDF file includes:

Main Text

Figures 1 to 2

Date: 10/27/2020 


\title{
Differential privacy will affect our understanding of trends in population growth in the United States
}

\begin{abstract}
The implementation of a proposed differential privacy algorithm to 2020 US Census data releases, and other census products has brought about discussions about the consistency and reliability of the data produced under the proposed disclosure avoidance system. We test the potential impact of this change in disclosure avoidance systems to the tracking of population growth and distribution using county-level population counts. We ask how population counts produced under the differential privacy algorithm might lead to different conclusions regarding population growth for the total population and three major racial/ethnic groups in comparison to counts produced using the traditional methods. Our results suggest that the implementation of differential privacy, as proposed, will impact our understanding of population changes in the US. We find potential for overstating and understating growth and decline, with these effects being more pronounced for non-Hispanic blacks and Hispanics, as well as for non-metropolitan counties. These findings draw attention to the potential local consequences of the implementation of differential privacy for tracking demographic changes of the US population, which is bound to have implications for our understanding of the transformations the nation is going through.
\end{abstract}




\section{How differential privacy will affect our understanding of trends in population growth in the United States}

\section{Introduction}

In September 2018, the US Census Bureau announced that differential privacy (DP) would be used to protect the 2020 census data (Mervis, 2019), officially replacing the traditional methods that included record-swapping, item imputation, household imputation, rounding, and top- and bottom-coding (Zayatz, 2007). In October 2019, the US Census Bureau released the first demonstration product that contained population counts produced under the proposed disclosure avoidance system (DAS) and enabled researchers to compare them with those produced under the traditional methods (U.S. Census Bureau, 2019). Numerous organizations, local government officials, and researchers have expressed concern about the implementation of DP to 2020 decennial census counts, and the utility of this data (Committee on National Statistics, 2020; Mervis, 2019). The changes in population counts, resulting from the implementation of DP, are so drastic that it could make small towns disappear (Wezerek \& Van Riper, 2020). For instance, the Committee of National Statistics sponsored a workshop where data users showed how the 2010 population counts differed from 2010 DP counts and the implications for their lines of inquiry in areas such as equity, political representation, and uses of these data in the public and private sector (Committee on National Statistics, 2020).

Analyses of the 2010 Demonstration Data Products have focused on studying the differences between 2010 counts produced under the traditional and currently proposed DAS, and have concluded that the differences are nontrivial. A recent analysis has concluded that the implementation of the initial algorithm would have affected our understanding in health disparities in the U.S. and a subsequent reanalyses did not render a different conclusion (Santos-Lozada, Howard, \& Verdery, 2020). Despite the growing body of analyses, no previous study has explored how this change will affect our understanding of population growth or decline across the US, and whether it will differently affect our understanding of this phenomenon across racial/ethnic groups and spatial categorizations.

\section{Materials and Methods}

Using the American Fact Finder, we accessed table Hispanic or Latino by Race (P007) and extracted county-level counts for four populations of interest: total population, non-Hispanic whites, non-Hispanic blacks and Hispanics from the 2000 decennial count (U.S. Census Bureau, 2001). We matched this data with the 2010-demonstration product published by the US Census on September 17, 2020, which contains corresponding counts for 2010 produced using two disclosure avoidance algorithms: 1. traditional disclosure avoidance practices and 2. the proposed differential privacy (Van Riper, Kugler, \& Schroeder, 2020). Traditional disclosure avoidance practices include geographic thresholds, rounding, noise addition, categorical thresholds, top coding, and data swapping (Zayatz, 2007). We calculate percent population 
change between 2000 and 2010 using the counts from the traditional disclosure methods $\left(P_{1}\right)$ and the demonstration products $\left(P_{2}\right)$. In Figure 1 , we compare $P_{1}$ and $P_{2}$ to assess differences in direction and magnitude of population change under both disclosure avoidance methods for the overall population and by race/ethnicity. To analyze spatial differences, we accessed the 2013 Rural-Urban Continuum codes (RUCC) published by the USDA (USDA, 2013). We matched each county with its corresponding RUCC and classified them as metropolitan or non-metropolitan. We present metro/non-metro differences with the color of each point in Figure 1. We calculate rate ratios for percent population change using the following formula: $R R=P_{1} / P_{2} \times 100$. In Figure 2, we present maps of the RR using five classifications: $<90,90-99$, 99-101, 101-110 and $>110$. Finally, we determined the percent of the counties, by state, that exhibited a displacement in population change by $10 \%$ and plotted it against population size (Figure 3 ).

\section{Results}

\section{Comparison of 2000-2010 Population Change under traditional and currently proposed disclosure avoidance systems}

Fig 1. compares the percent change in population between 2000 and 2010 when counts produced under both disclosure avoidance systems are compared to 2000 census counts. We find that percent changes for the overall population and non-Hispanic whites are similar when we compare indicators produced using the 2010 counts derived from both disclosure avoidance systems (Panels $A-B$ ). We find differences in population change between 2000 and 2010 using the different DAS for non-Hispanic blacks and Hispanics (Panels C-D). That is, county-level population growth is consistent for the overall population and nonHispanic whites when comparing both DAS, but this is not the case for non-Hispanic blacks and Hispanics. We found that $95.79 \%$ and $78.87 \%$ of the counties had comparable population change trajectories for the overall population and non-Hispanic whites, respectively. On the other hand, the counties with comparable population change was $42.09 \%$ and $63.06 \%$ for non-Hispanic blacks and Hispanics, respectively. Each panel in Fig. 1 includes a distinction for metropolitan and non-metropolitan counties, represented as red and blue respectively. We found no difference by metropolitan classification for the overall population nor for non-Hispanic whites. On the other hand, we found population change indicators for non-Hispanic blacks and Hispanics to be more variable in non-metropolitan areas (blue). 

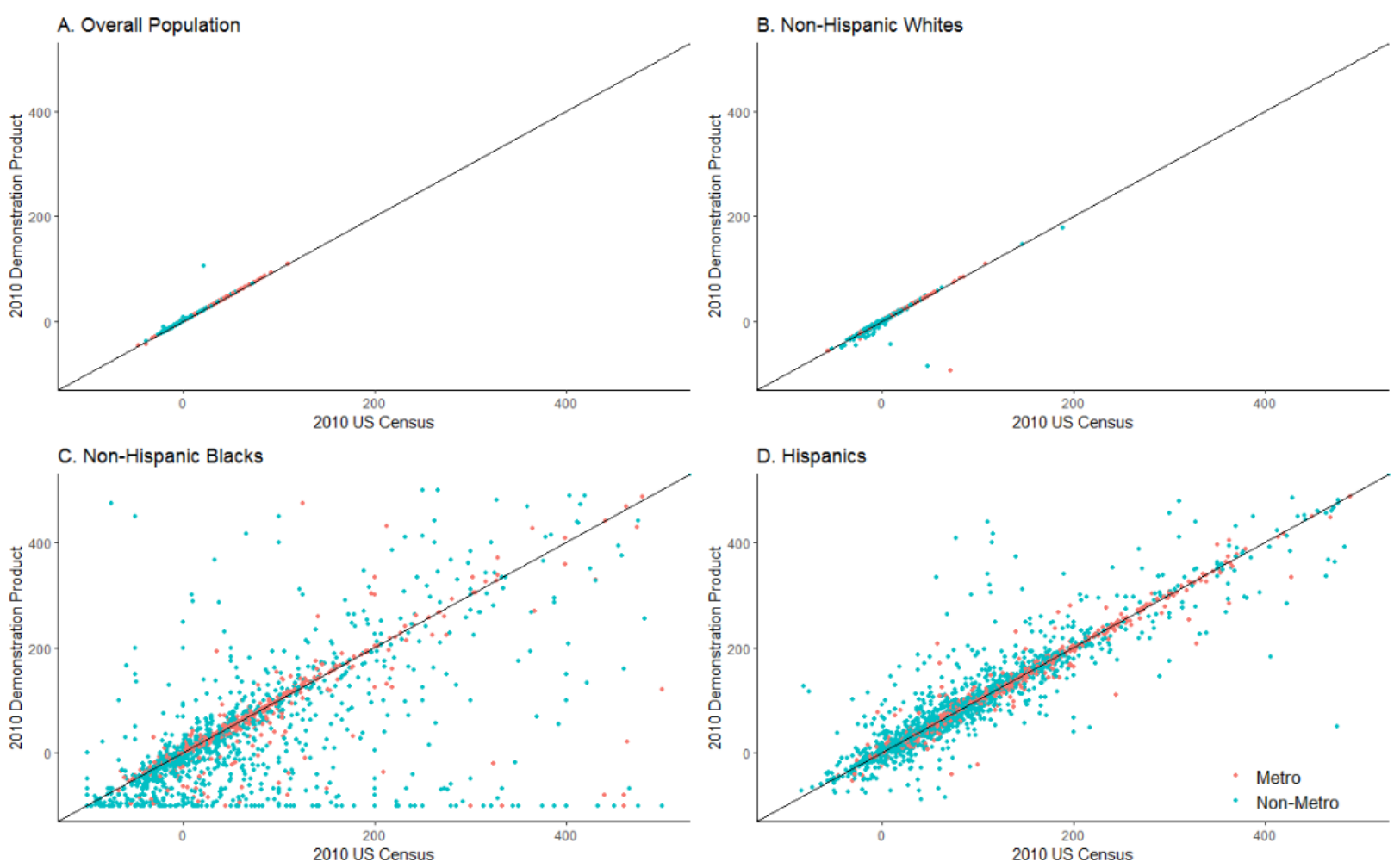

Figure 1. Percent Population Change 2000-2010 comparing 2010 counts produced under traditional disclosure avoidance practices and with the implementation of the proposed differential privacy algorithm colored by Metropolitan/Non-Metropolitan classification.

Figure 2, presents maps of the rate ratios (RR) of percent population change between 2000 and 2010, using the 2010 counts produced using the traditional $\left(P_{1}\right)$ and the proposed DAS $\left(P_{2}\right)$. We find that counties with RR that are below 90 or over 100 , a difference of higher than $10 \%$ in both directions, to be 132 for the overall population (4.21\%), 663 for non-Hispanic whites (21.13\%), 1,725 for non-Hispanic blacks (57.91\%), and 1,154 for Hispanics (36.94\%). This is indicative that latest revision of DP, as proposed, would continue to affect population change metrics not only for non-Hispanic blacks and Hispanics, but also for nonHispanic whites. The population counts that are less affected are those for the overall population. 

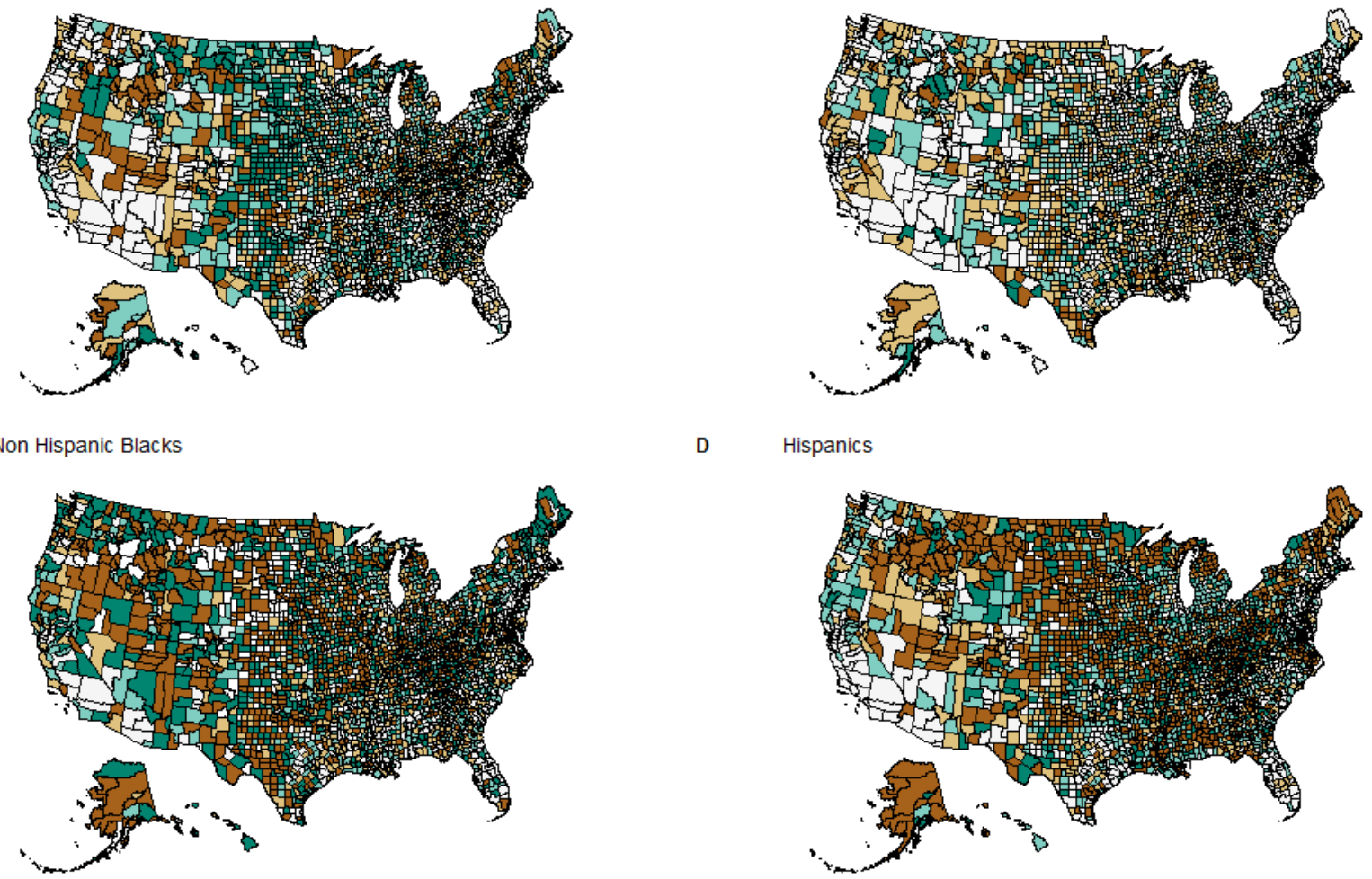

Change Categories

1. Below 90 2. Between 90-99 $\square$ 3. Between 99-101 4. Between 101.1-110 5. Over 110

Figure 2. Rate Ratios for Percent Population Change comparing 2010 counts produced under traditional disclosure avoidance methods and the differential privacy demonstration products.

In Figure 3, we present illustrate the percent of the counties that have a population growth displacement (error) over $10 \%$. For the overall population we found that these differences in percent change of $10 \%$ or more were not present in Alabama, Arizona, Connecticut, Washington D.C., Delaware, Florida, Massachusetts, Maryland, Maine, New Hampshire, New Jersey, Rhode Island, Utah and Vermont. Connecticut, Delaware, and New Jersey. For the non-Hispanic white population, we did not find counties with a displacement in population growth over $10 \%$ in Connecticut, Washington D.C., Delaware, and Rhode Island. The most affected states were Idaho and Alaska, with $37 \%$ and $38 \%$ of their counties exceeding $10 \%$ variation in population change indicators. For non-Hispanic blacks, we found no difference in percent change over $10 \%$ for Washington D.C., Delaware, Hawaii, New Jersey and Rhode Island. We found that 14 states had more than $50 \%$ of the counties with percent changes over $10 \%$. For Hispanics, we found that Connecticut, Washington D.C., Delaware, Hawaii, Massachusetts, New Hampshire, New Jersey and Rhode Island did not have any percent change over $10 \%$. In contrast, 14 states had more than half of their counties with a percent change over $10 \%$. Eleven states had more than $50 \%$ of their counties exceeding the $10 \%$ threshold in population change metrics for Hispanics. We note a negative correlation between the percent of the counties with $10 \%$ or higher error in minority counts (Panels $C$ and $D$ ), and population size. 

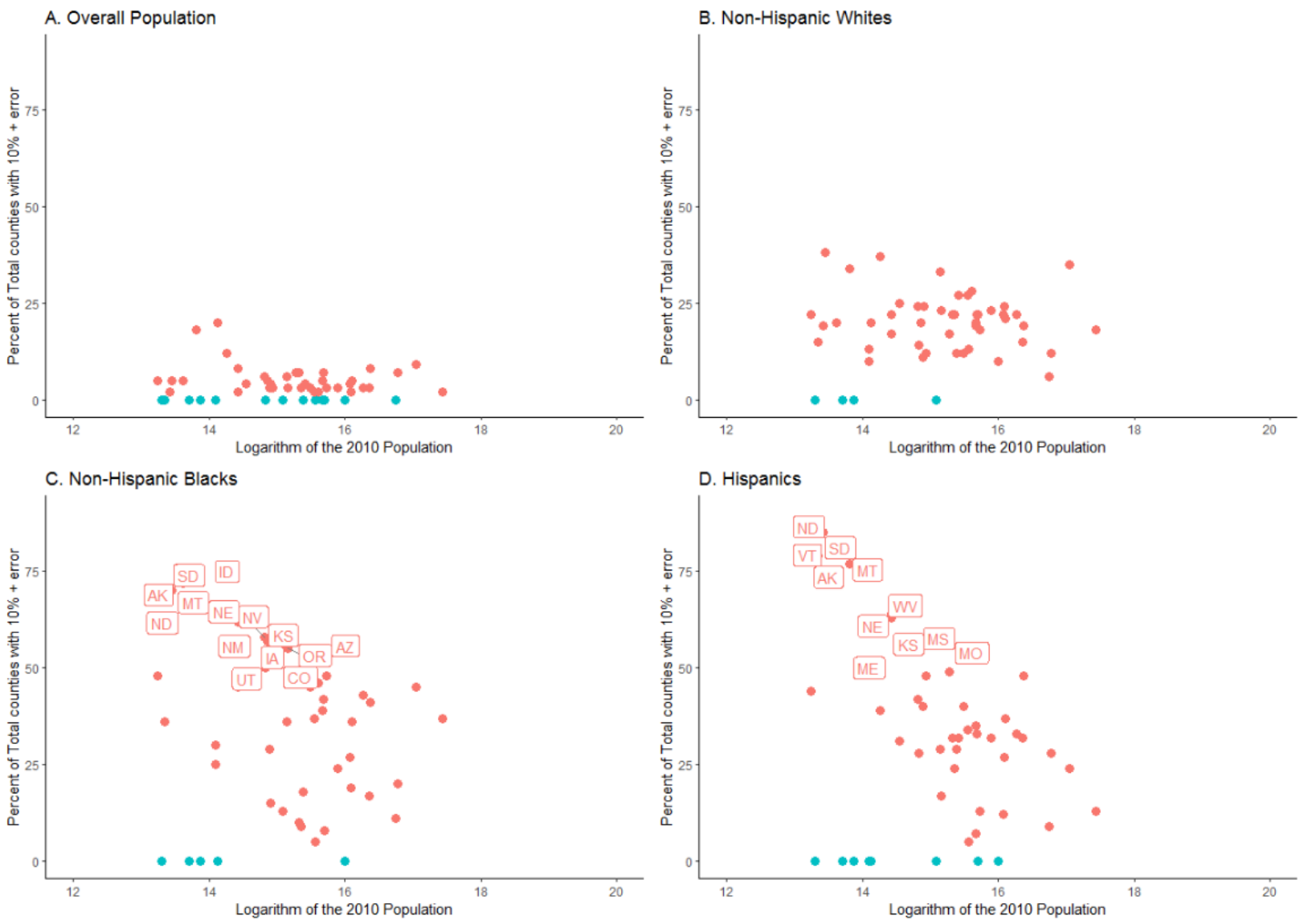

Figure 3. Percent of the counties with more than $10 \%$ displacement in population growth/decline by population size. States with more than $50 \%$ of the counties exhibiting displacements over $10 \%$ include the abbreviation. In blue, we include states with zero counties where displacement in population change occurred above $10 \%$.

\section{Conclusion and Discussion}

The decennial census is the principal source of statistical information in the United States. The uses of these tabulations have grown out of the purpose as detailed in the US Constitution. The implementation of DP, as proposed, to decennial tabulation would limit our understanding of demographic changes happening in the United States. It is troubling that these changes are more pronounced for racial/ethnic minorities.

Future DP proposals should be mindful of these substantial changes observed in the data, even when the algorithm has been revised numerous times. In addition, these changes will have implications concerning our understanding of population change; this will undoubtedly affect our tracking of the demographic transformations occurring in the US. Subsequent considerations should include the implications for the public and the private sectors who rely on the US Census data as a crucial source of information. The instances where the implementation of the currently proposed DAS include but are not limited to federal and local redistricting, decision-making that relies on demographic data, and tracking of health incidence and prevalence by sectors of the population. 
In addition, changes in the DAS should also consider the implications of this change for analyses of population indicators across time. We demonstrate that 2010 counts produced using DP alters decennial change calculations in population, with change estimates being more prominent for racial/ethnic minorities.

\section{Acknowledgments}

We thank Reviewer \#2 who suggested this analysis during a previous round of reviews. We thank the IPUMS and NHGIS teams for sharing the 2010 demonstration products in a format accessible for the data user community. This work was supported by the Population Research Institute (Grant P2CHD041025), the Data Accelerator, and the Social Science Research Institute at the Pennsylvania State University. We thank David Van Riper for his support in the early stages of this work. We accessed 2000 counts through American Factfinder, with this paper we say farewell to this friend of the data-user community.

\section{Reference}

Committee on National Statistics. (2020). Workshop on 2020 Census Data Products: Data Needs and Privacy Considerations. In Videos and Presentations from the Workshop. Washington, DC: National Academies of Sciences, Engineering and Medicine. Retrieved from https://sites.nationalacademies.org/DBASSE/CNSTAT/DBASSE_196518

Mervis, J. (2019). Can a set of equations keep U.S. census data private? Science. https://doi.org/10.1126/science.aaw5470

Santos-Lozada, A. R., Howard, J. T., \& Verdery, A. M. (2020). How differential privacy will affect our understanding of health disparities in the United States, 1-8. https://doi.org/10.1073/pnas.2003714117

U.S. Census Bureau. (2001). 2000 Census of Population and Housing. U.S. Department of Commerce, Economics and Statistics Administration.

U.S. Census Bureau. (2019). 2010 Demonstration Data Products. Retrieved January 1, 2019, from https://www.census.gov/programs-surveys/decennial-census/2020-census/planningmanagement/2020-census-data-products/2010-demonstration-data-products.html

USDA. (2013). Rural-Urban Continuum Codes. Retrieved February 2, 2020, from https://www.ers.usda.gov/data-products/rural-urban-continuum-codes.aspx

Van Riper, D., Kugler, T., \& Schroeder, J. (2020). IPUMS NHGIS Privacy-Protected 2010 Cesus Demonstration Data, version 20200917 [Database]. Minneapolis, MN: IPUMS.

Wezerek, G., \& Van Riper, D. (2020, February 6). Changes to the Census Could Make Small Towns Disappear. New York Times. Retrieved from https://www.nytimes.com/interactive/2020/02/06/opinion/census-algorithm-privacy.html

Zayatz, L. (2007). Disclosure avoidance practices and research at the U.S. Census Bureau: an update. Journal of Official Statistics, 23(2), 253-265. 